\title{
Fatores condicionantes da adesão dos alunos ao Programa de Alimentação Escolar no Brasil ${ }^{1}$
}

\author{
Conditioning factors of students participation in the \\ Brazilian School Meal Program
}

\author{
Gilma Lucazechi STURION² \\ Marina Vieira da SILVA² \\ Ana Maria Holland OMETTO ${ }^{2}$ \\ Maria Cristina Ortiz FURTUOSO² \\ Maria Angélica Penatti PIPITONE ${ }^{3}$
}

\section{R E S U M O}

\section{Objetivo}

Verificar o nível de adesão dos alunos ao Programa de Alimentação Escolar e identificar as principais variáveis que a afetam.

\section{Métodos}

A pesquisa baseou-se em amostra composta por dez municípios brasileiros, sendo dois de cada região geográfica. As informações foram obtidas a partir de visitas às escolas (duas por município) e entrevistas junto a 2678 escolares. Foram consideradas no modelo estatístico as variáveis: gênero, idade, práticas alimentares, estado nutricional e condições socioeconômicas dos alunos. Utilizaram-se os testes de qui-quadrado e o modelo de lógite para identificar a influência simultânea das variáveis acima consideradas.

\section{Resultados}

Verificou-se que $46 \%$ dos alunos consomem diariamente a alimentação oferecida na escola, enquanto $17 \%$ não participam do Programa. As variáveis renda familiar per capita, escolaridade dos pais, idade, estado nutricional dos alunos e a maior freqüência de consumo de alimentos nas "cantinas escolares", ou seja, cantinas comerciais existentes nas escolas, são inversamente associadas à adesão diária ao Programa. Nas escolas que não dispõem de tais cantinas, o estado nutricional é a única variável inversamente associada à adesão diária ao Programa.

\footnotetext{
1 Artigo elaborado a partir de tese de G.L. Sturion intitulado "Programa de Alimentação Escolar: avaliação de desempenho em dez municípios brasileiros". Faculdade de Engenharia de Alimentos, Universidade Estadual de Campinas. Campinas, 2002. 269p.

2 Departamento de Agroindústria, Alimentos e Nutrição, Escola Superior de Agricultura Luiz de Queiroz, Universidade de São Paulo. Av. Pádua Dias, 11, 13418-900, Piracicaba, SP, Brasil. Correspondência para/Correspondence to: G.L.STURION. E-mail: <glsturio@esalq.usp.br>.

3 Departamento de Economia, Administração e Sociologia, Escola Superior de Agricultura Luiz de Queiroz, Universidade de São Paulo. Piracicaba, SP, Brasil.
} 


\section{Conclusão}

Pode-se inferir que a adesão ao Programa de Alimentação Escolar é baixa e que, embora concebido para ser universal, na prática, assume caráter focalizado, beneficiando principalmente os escolares comprometidos nutricionalmente, cujas famílias possuem menores rendimentos e escolaridade. Espera-se que o presente estudo possa contribuir para a reformulação do Programa, visando promover a adequação do seu atendimento às características dos beneficiários. E que possa, também, subsidiar pesquisas futuras para identificar os motivos da rejeição da alimentação escolar por expressiva parcela da população alvo.

Termos de indexação: alimentação escolar, estudantes, política pública, programa de alimentação e nutrição.

\section{A B S T R A C T}

\section{Objective}

The main objectives of this research are to verify the level of students' participation in the National School Meal Program in Brazil, and to identify the factors that influence their adherence to the program.

\section{Methods}

The research was based on a sample conformed by ten Brazilian municipalities, being localized, each two of them in one of the five geographic regions. Data were collected in the schools and interviews were conducted with 2678 students. Students' gender, age, feeding practices, nutritional status, and socioeconomic conditions were taken into account by the adopted statistical model. The chi-square test and logit model were employed as means to identify simultaneous influences of the factors involved.

\section{Results}

It was verified that $46 \%$ of the students consumed the school meals on a daily basis, whereas $17 \%$ didn't participate in the Program. Such factors as per capita family income, level of educational of parents, student's nutritional status and frequency of food consumption at the commercial establishment at school are contrariwise associated to the daily Program participation. Nutritional status was the only factor inversely associated with daily adherence to the Program in schools where no commercial food establishment was available.

\section{Conclusion}

It may well be concluded that the participation in the Brazilian National School Meal Program is low and, although designed to be universal in scope, in practice, it appears to be restricted, benefiting mostly students with nutritional status at risk, from families of lower income and educational levels. It is expected that the present study will contribute to program reforms, adapting it to a wider range of characteristics, which should be more appealing to the potential beneficiaries. Additionally, these data should afford the basis for future researches, in order to identify the causes of program rejection by a substantial portion of the students.

Indexing terms: school feeding, students, public policies, food and nutrition program.

\section{N T R O D U Ç Ã O}

Por meio da distribuição de refeições durante o intervalo das atividades escolares, o Programa Nacional de Alimentação Escolar (PNAE) visa suplementar a alimentação do aluno, melhorando suas condições nutricionais e sua capacidade de aprendizagem. Objetiva, também, formar bons hábitos alimentares¹.

Assegurado pela Constituição de 1988, o PNAE tem caráter universal e atendeu, em 2002, aproximadamente 36 milhões de alunos da pré-escola e do ensino fundamental da rede pública e filantrópica de ensino, com investimentos na ordem de $\mathrm{R} \$ 849$ milhões ${ }^{1}$.

Mesmo ocupando um lugar de destaque entre as políticas sociais, esse Programa não tem sido alvo de avaliações contínuas, que permitiriam reorientá-lo, reformulá-lo ou mesmo reforçá-lo, de maneira a otimizar os recursos utilizados.

Implementado até 1993 de forma centralizada no governo federal, o Programa apresentava todo tipo de distorções, dentre as quais, a inadequação de cardápios quanto à 
qualidade nutricional e atendimento aos hábitos alimentares, além da irregularidade no oferecimento da alimentação ${ }^{2,3}$. Acrescente-se a isso a precariedade do Programa nas regiões mais pobres e a baixa adesão dos escolares. A Pesquisa Nacional de Nutrição e Saúde (PNSN), realizada em $1989^{4}$, revelou que somente $78,0 \%$ dos alunos freqüentavam escolas que ofereciam merenda ${ }^{5}$, sendo que a maioria dessas situavam-se nas regiões mais ricas do país ${ }^{6}$.

Nesse sentido, essa pesquisa evidenciou que as Regiões Sul e Sudeste possuíam a maior proporção de crianças e jovens freqüentando unidades que ofereciam merenda escolar, embora essa população apresentasse a menor incidência de desnutrição crônica ${ }^{7}$. Evidenciou, ademais, que o percentual de crianças desnutridas beneficiadas nas áreas rurais $(63,4 \%)$ era nitidamente inferior ao das áreas urbanas $(82,8 \%)$, sendo que o alcance do PNAE na área rural do Nordeste era ainda mais restrito. Tais dados reforçaram o argumento das distorções, especialmente no que tange às desigualdades regionais ${ }^{8}$. Quanto ao consumo efetivo dos alimentos distribuídos pelo Programa, os dados da PNSN mostraram que, pouco mais de $40,0 \%$ dos alunos consumiam todos os dias a refeição oferecida, sendo que os índices de consumo se reduziam com o aumento da renda familiar; ademais, evidenciou-se que, mesmo entre os alunos mais pobres, a participação no Programa era baixa, ou seja, em torno de $57,0 \%$.

Operacionalizado de forma descentralizada desde 1994, o Programa é atualmente coordenado nacionalmente pelo Fundo Nacional de Desenvolvimento da Educação (FNDE), do Ministério da Educação e do Desporto (MEC). O referido órgão é responsável pelo repasse dos recursos financeiros para aquisição de alimentos, cabendo aos Estados e Municípios complementar estes recursos além de cobrir os custos operacionais.

Com a descentralização, mudanças significativas têm sido observadas na operacionalização do Programa, que se refletem na melhoria da qualidade dos serviços. Alterações na sistemática de compras, implantação de produção alternativa de alimentos e utilização de maior volume de alimentos básicos e in natura foram os procedimentos alternativos incorporados ao Programa, especialmente na última década ${ }^{9-11}$.

No entanto, ainda há escassez de informações que possibilitem uma completa avaliação do Programa após a descentralização. As pesquisas voltadas à análise da sua eficácia são isoladas e, predominantemente, realizadas no Estado de São Paulo, as quais, entretanto, também revelam existir nesse estado um nível de participação aquém do esperado entre os beneficiários potenciais do Programa12,13.

Com base nessas considerações, o presente trabalho tem o objetivo de verificar o nível de adesão dos escolares ao Programa e identificar as principais variáveis que a afetam, de modo a refletir mais adequadamente a realidade nacional.

\section{MÉ T O D OS}

Os dados analisados neste trabalho integram pesquisa de natureza quali-quantitativa, com o objetivo de conhecer o desempenho do PNAE em municípios com diferentes modalidades de descentralização e/ou vinculados aos programas compensatórios e/ou de qualidade ${ }^{14}$. A coleta dos dados foi implementada durante os meses de setembro e novembro de 1997.

Destaca-se que a referida pesquisa baseou-se em uma amostra de conveniência, composta por dois municípios selecionados (um de porte mediano e um de grande porte) de um estado de cada grande região brasileira, perfazendo um total de dez, que contemplavam as especificidades de implementação do Programa requeridas no estudo.

Para a seleção dos municípios considerou-se a população estimada, em 1995, de cada um deles ${ }^{15}$. Os municípios de porte mediano foram identificados para cada um dos cinco estados previamente selecionados, a partir de um software específico. Os municípios de grande porte foram 
selecionados sempre entre os três maiores, excluindo-se a capital do respectivo estado.

Os municípios integrantes da amostra foram: Parnaíba (127 929 habitantes) e Brasileira (8 162 habitantes), do Estado do Piauí, PI, Abaetetuba (99 989 habitantes) e Tailândia (17 707 habitantes), do Estado do Pará, PA, Contagem (449 588 habitantes) e Baldim (8 383 habitantes), do Estado de Minas Gerais, MG, Anápolis (239 378 habitantes) e Itaguarú (6 450 habitantes), do Estado de Goiás, GO e Joinville (347 151 habitantes) e Ponte Serrada (12 259 habitantes), do Estado de Santa Catarina, SC.

A coleta de informações foi realizada por pesquisadores com formação universitária, na área de alimentação e nutrição, previamente treinados. O processo de obtenção dos dados envolveu visitas a duas unidades de ensino de cada município selecionado. As referidas unidades (localizadas em diferentes regiões do município e indicadas pelos gerentes municipais do Programa) eram, em sua maioria, de porte médio (de 150 a 500 alunos matriculados) e grande (mais de 500 alunos matriculados), de acordo com a classificação do $M E C^{15}$, pertenciam à rede pública de ensino, e atendiam crianças de 7 a 14 anos no período diurno.

Os instrumentos metodológicos adotados foram: formulário para o registro dos dados antropométricos (peso e altura), questionário aplicado pelos entrevistadores para obtenção das informações junto aos alunos e questionário auto-administrado para os pais dos alunos amostrados.

Os questionários foram estruturados objetivando identificar a freqüência de participação dos escolares no PNAE e as variáveis que poderiam afetá-la, tais como idade, gênero, práticas alimentares (freqüência semanal de compra de alimentos na cantina escolar e número de refeições realizadas no domicílio) e estado nutricional dos alunos. Procurou-se, também, identificar o estrato socioeconômico do escolar, a partir da renda familiar, número de pessoas que viviam no domicílio e escolaridade dos pais, além da presença de cantinas escolares, ou seja, estabelecimentos que comercializam alimentos na unidade de ensino.

Cabe registrar que, visando definir a metodologia a ser adotada para a obtenção dos dados, a avaliação do desempenho dos técnicos e o melhor procedimento operativo, realizou-se um pré-teste em 3 municípios do Estado de São Paulo (com características socioeconômicas semelhantes às dos municípios que constituíam a amostra definitiva).

A partir desse pré-teste, foi possível definir o número de alunos que integrariam a amostra (o tempo demandado para cada entrevista foi um dos critérios considerados), sendo selecionados, em cada escola, 100 alunos de 1a a $4^{a}$ séries (faixa etária esperada de 7 a 10 anos), com 25 em cada série, e 52 alunos de 5 a a 8 a séries (faixa etária esperada de 11 a 14 anos), com 13 crianças em cada série.

No total, foram entrevistados 2678 alunos, sendo $73,0 \%$ com idade entre 7 a 10 anos e $27,0 \%$ com idade acima de 11 anos. A inclusão de um menor número de alunos na amostra de 5 a

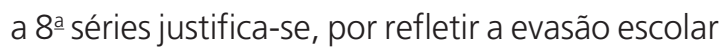
historicamente observada no país. Quanto à representação de alunos por gênero, a amostra constituiu-se de $55,3 \%$ de meninas e $44,1 \%$ de meninos.

Para a avaliação do estado nutricional, obteve-se o peso e a altura (tomadas no âmbito da escola) de acordo com os procedimentos recomendados pela World Health Organization ${ }^{16}$.

A partir do registro das medidas antropométricas, da idade (obtida na entrevista com os escolares e confirmada por meio de consulta aos documentos disponíveis nas secretarias das escolas) e do sexo, analisou-se a distribuição dos percentis do índice de massa corporal (IMC) ou Índice de Quetelet, obtido por meio da expressão $\mathrm{IMC}=$ peso $(\mathrm{kg}) /[\text { altura }(\mathrm{m})]^{2}$.

Adotou-se como referência, as curvas propostas pelo Nacional Center for Health Statistics (NCHS) em 2000 17 para distribuição dos percentis 
do IMC de acordo com idade e sexo dos alunos e os seguintes níveis críticos: IMC $<5$ 우 como indicativo de desnutrição; IMC < 10P como indicativo de baixo peso; $85^{\circ} \leq \mathrm{IMC}<95^{\circ} \mathrm{P}$ como indicativo de sobrepeso e IMC $\geq 95$ op como indicativo de obesidade.

Para viabilizar os cálculos da distribuição dos percentis e a respectiva comparação com o padrão de referência foi desenvolvido um software específico.

Para a elaboração das análises estatísticas, foi construído um banco de dados, utilizando-se o software Fox-base (com posterior transformação em D-Base), compatível com o software adotado (Statistical Analytical System - SAS - versão 8 de 1999).

Elaboraram-se tabelas de contingência acompanhadas dos testes de qui-quadrado comum, destinadas a captar qualquer tipo de relação entre duas variáveis utilizadas e o qui-quadrado de tendência linear de MantelHaenszel $^{18}-\chi^{2} \mathrm{MH}$. Este último destina-se a detectar a existência de tendência de crescimento ou diminuição da proporção de uma variável dependente, em função do nível da outra variável considerada.

Para identificar o efeito das variáveis na adesão dos escolares ao Programa de Alimentação Escolar foi adotado o modelo de lógite. Este modelo estatístico permite avaliar, simultaneamente, a influência de diversas variáveis explanatórias sobre uma variável dependente, quando esta é uma proporção. O coeficiente de cada variável explanatória, à semelhança de uma regressão múltipla, indica a sua influência sobre a dependente, descontado o efeito das demais variáveis explanatórias incluídas no modelo.

Foram definidos dois modelos com variáveis dependentes distintas e variáveis explanatórias comuns. Um dos modelos analisou como variável dependente "a participação do aluno no Programa"; o outro, avaliou como variável dependente "a freqüência semanal de consumo da alimentação escolar".
Considerando-se, também, que somente nove unidades escolares possuíam cantina escolar, definiram-se os referidos modelos tendo por base dois grupos: um, constituído pelos alunos pertencentes às unidades de ensino que não possuíam cantina escolar; o outro, pelos alunos pertencentes às escolas que mantinham esse tipo de estabelecimento incluindo, neste último, a variável explanatória, "freqüência semanal de consumo de alimentos na cantina escolar" (alimentos comprados pelo aluno no estabelecimento comercial dentro da escola).

A variável dependente "participação do aluno no Programa" assume o valor 1, quando a resposta do escolar é afirmativa, e valor zero, quando negativa. Neste caso, empregou-se o modelo de lógite para variável binária, uma vez que essa variável dependente envolve duas categorias de respostas.

A variável dependente "freqüência semanal de consumo da alimentação escolar" apresenta três categorias de respostas (uma vez por semana, duas a três vezes por semana e quatro ou cinco vezes por semana) que envolvem ordenação, da menor para a maior freqüência semanal de consumo. Por isso, para essa variável, empregou-se o modelo de lógite multinomial cumulativo, de acordo com Allison ${ }^{19}$.

Antecedendo a definição dos modelos de lógite, visando a verificação da existência de colinearidade, efetuou-se teste por meio de análise de estrutura (índice de condicionamento e proporção de variação) de todas as possíveis variáveis que deveriam integrar os modelos ${ }^{20}$.

Uma vez confirmada a inexistência de colinearidade, efetuou-se a seleção de variáveis para definição do modelo por meio da adoção do método stepwise. Esse método prevê, inicialmente, a identificação da variável explanatória que produz um modelo ótimo para uma variável dependente. Na seqüência, é incorporada outra variável que produz a maior redução na soma de quadrados dos resíduos (maior acréscimo no $R^{2}$ ). Após a inclusão de cada variável, a equação resultante é examinada, com o objetivo de 
identificar se qualquer coeficiente tem um valor $p$ suficientemente elevado, o que sugere que a variável deva ser excluída do modelo. O processo tem continuidade, até que nenhuma adição ou exclusão de variáveis explanatórias seja recomendada, tendo como critério os níveis de significância estabelecidos ${ }^{20}$.

Destacam-se dois tipos de variáveis explanatórias comuns aos dois modelos: a) binárias: idade $(0=$ escolar de 7 a 10 anos e $1=$ escolar de 11 anos ou mais) e gênero ( 0 = masculino e 1 = feminino); b) contínuas: renda familiar per capita (valores em reais); escolaridade do pai e da mãe (anos de estudo); estado nutricional (percentil do IMC) e número de refeições que o aluno recebe no domicílio.

Para o grupo de alunos pertencentes às escolas que possuíam cantina escolar, a variável explanatória "freqüência semanal de consumo de alimentos da cantina escolar", foi incluída no modelo, indicando o número de vezes por semana que $o$ aluno respondeu adquirir alimentos no estabelecimento.

O modelo de lógite produz estimativas de probabilidade que variam entre 0 e 1 . Pode ser representado pela seguinte equação:

na final:

$$
P=\frac{1}{1+e^{-z}}
$$

$P=$ probabilidade de um aluno com determinadas características consumir a alimentação escolar.

Nos dados observados considerou-se uma variável binária que, conforme mencionado, assume o valor 1 quando o aluno responde que participa do Programa, ou valor zero, em caso contrário.

$e=$ constante $=2,71828$

$Z$ é o lógite, que é uma função linear das variáveis explanatórias: $Z=\alpha+\Sigma \beta_{i} X_{i}=\alpha+\beta_{1} X_{1}+\beta_{2} X_{2}$ $+\ldots+\beta_{k} X_{k}$ os termos $X$ são as variáveis explanatórias de interesse e assumem os valores obtidos na pesquisa; os termos $\alpha$ e $\beta$ são os parâmetros estimados pelo método "máxima verossimilhança", a partir dos dados obtidos na pesquisa.
Para o modelo de lógite multinomial cumulativo as fórmulas são as seguintes:

$\ln \frac{P_{1}}{1-P_{1}}=\alpha_{1}+\sum \beta_{1} X_{1}$ In $\frac{P_{2}}{1-P_{2}}=\alpha_{2}+\sum \beta_{1} X_{1}$ na final:

$\mathrm{P}_{1}=$ probabilidade do aluno consumir a alimentação escolar 1 dia por semana;

$\mathrm{P}_{2}=$ probabilidade do aluno consumir a alimentação escolar 1, 2 ou 3 vezes por semana; $1-\mathrm{P}_{2}=$ probabilidade do aluno consumir $\mathrm{a}$ alimentação escolar 4 ou mais vezes por semana.

\section{RESULTADOSE DISCUSSÃO}

Integravam os questionários aplicados aos alunos, duas questões relativas ao consumo da alimentação escolar. A primeira registrava "Você costuma comer a alimentação oferecida na escola?". O aluno deveria escolher entre duas alternativas propostas, "Sim" ou "Não". A segunda questão, "Se você respondeu que come a alimentação escolar, marque quantos dias por semana: uma vez por semana, duas a três vezes por semana ou quatro a cinco vezes por semana". Nesse caso, o aluno deveria marcar somente uma das três alternativas propostas.

A partir das respostas dos entrevistados, elaborou-se a Tabela 1, na qual estão reunidos os dados referentes à freqüência de consumo da alimentação escolar pelos alunos no âmbito da unidade de ensino.

A análise dos dados revela que a adesão diária média é baixa, se considerarmos como efetiva adesão ao Programa o consumo de quatro ou cinco vezes por semana da alimentação oferecida na escola. Destaca-se que, com essa freqüência, somente em nove unidades escolares, a maioria dos alunos aderiam ao Programa. Tal realidade foi observada em algumas unidades de ensino dos Estados do Piauí, Pará e Goiás. Destaca-se, também, a situação observada no município de Joinville, SC, onde substancial parcela dos alunos de uma escola (17GG-SC) afirmou não consumir a alimentação oferecida. 
Tabela 1. Distribuição dos escolares* de acordo com a freqüência de consumo da alimentação escolar e unidade de ensino, 1997.

\begin{tabular}{|c|c|c|c|c|c|c|c|c|}
\hline \multirow[b]{2}{*}{ Unidade de ensino** (semanas) } & \multicolumn{2}{|c|}{ Não consome } & \multicolumn{2}{|c|}{$1 \mathrm{vez}$} & \multicolumn{2}{|c|}{2 a 3 vezes } & \multicolumn{2}{|c|}{4 a 5 vezes } \\
\hline & $\mathrm{n}$ & $\%$ & $\mathrm{n}$ & $\%$ & $\mathrm{n}$ & $\%$ & $\mathrm{n}$ & $\%$ \\
\hline $1 \mathrm{GM}-(\mathrm{PI})$ & 4 & 4,4 & 8 & 8,9 & 15 & 16,7 & 61 & 67,8 \\
\hline $2 \mathrm{GG}-(\mathrm{PI})$ & 26 & 18,2 & 11 & 7,7 & 42 & 29,4 & 64 & 44,8 \\
\hline 3MM-(PI) & 6 & 4,1 & 6 & 4,1 & 36 & 24,7 & 100 & 68,5 \\
\hline $5 G G-(P A)$ & 57 & 37,3 & 21 & 13,7 & 43 & 28,1 & 31 & 20,3 \\
\hline $6 \mathrm{GM}-(\mathrm{PA})$ & 13 & 13,0 & 9 & 5,9 & 24 & 24,0 & 53 & 53,0 \\
\hline 7MG-(PA) & 23 & 14,8 & 10 & 10,0 & 41 & 26,5 & 84 & 54,2 \\
\hline 8MG-(PA) & 21 & 15,7 & 14 & 9,0 & 19 & 14,2 & 79 & 59,0 \\
\hline 9GG-(MG) & 41 & 27,7 & 22 & 16,4 & 59 & 39,9 & 29 & 19,6 \\
\hline $10 G G-(M G)$ & 35 & 23,2 & 20 & 13,5 & 63 & 41,7 & 34 & 22,5 \\
\hline $11 \mathrm{MM}-(\mathrm{MG})$ & 16 & 9,4 & 15 & 9,9 & 61 & 35,7 & 81 & 47,4 \\
\hline $12 \mathrm{MM}-(\mathrm{MG})$ & 4 & 5,3 & 0 & 0,0 & 43 & 56,6 & 29 & 38,2 \\
\hline $13 \mathrm{GG}-(\mathrm{GO})$ & 1 & 0,7 & 0 & 0,0 & 29 & 19,2 & 120 & 79,5 \\
\hline $14 \mathrm{GG}-(\mathrm{GO})$ & 3 & 2,0 & 1 & 0,7 & 22 & 14,4 & 124 & 81,0 \\
\hline 15MG-(GO) & 3 & 2,2 & 4 & 2,6 & 39 & 28,1 & 94 & 67,6 \\
\hline 16MM-(GO) & 1 & 0,7 & 4 & 2,9 & 35 & 24,3 & 104 & 72,2 \\
\hline $17 \mathrm{GG}-(\mathrm{SC})$ & 101 & 69,7 & 26 & 18,1 & 18 & 12,4 & 5 & 3,4 \\
\hline $18 G G-(S C)$ & 39 & 25,2 & 16 & 11,0 & 37 & 23,9 & 64 & 41,3 \\
\hline $19 \mathrm{MG}-(\mathrm{SC})$ & 48 & 31,8 & 27 & 17,4 & 53 & 35,1 & 23 & 15,2 \\
\hline 20MG-(SC) & 7 & 4,8 & 21 & 13,9 & 74 & 50,3 & 47 & 32,0 \\
\hline Total & 449 & 16,9 & 235 & 8,8 & 753 & 28,3 & 1226 & 46,0 \\
\hline
\end{tabular}

${ }^{*} \mathrm{n}=2$ 663; ** Código da escola: número designado pelos pesquisadores para identificação da escola, seguido pelas letras referentes ao porte do município e ao porte da escola, mais a sigla da unidade federativa a que pertence. ( $G=$ grande, $M=$ médio, P= pequeno); Pl= Piauí; $\mathrm{PA}=$ Pará; $\mathrm{MG}=$ Minas Gerais; $\mathrm{GO}=$ Goiás; $\mathrm{SC}=$ Santa Catarina. Escola número 4: dados não disponíveis.

É importante notar ainda que, comparando-se as proporções do consumo de alunos, observado em duas escolas de um mesmo município, foram encontradas situações bastante distintas, como em Abaetetuba, PA (5GG-PA e 6GM-PA) e Joinville, SC (17GG-SC e 18GG-SC).

Em pesquisa realizada pelo FNDE, em 2001, tendo por base amostra representativa de unidades escolares públicas do Brasil ${ }^{15}$, observou-se adesão diária ao Programa de 62,4\% dos alunos pertencentes às escolas urbanas. Embora a pesquisa tenha revelado percentuais superiores aos dados do presente trabalho, o que pode sugerir uma possível melhoria nos níveis de adesão no período compreendido entre as duas pesquisas, deve-se admitir que o Programa tem muitos desafios a enfrentar, no tocante a estimular e ampliar as adesões entre a população alvo.

Dentre as variáveis explanatórias relacionadas aos escolares, analisou-se a associação entre a faixa etária e o gênero dos alunos e as variáveis dependentes "participação dos alunos no Programa" e "freqüência semanal de consumo".

Foi verificada forte associação entre faixa etária e "participação dos alunos no Programa" ( $\chi^{2} \mathrm{MH}=98,6$, com um grau de liberdade, significativo a 1,0\%). Da totalidade da amostra $(n=2652), 87,5 \%$ dos alunos mais novos afirmaram que participavam do Programa, enquanto apenas $71,2 \%$ dos mais velhos revelaram o mesmo comportamento.

Embora os dados tenham evidenciado maior freqüência semanal de consumo entre os escolares de 7 a 10 anos de idade ( $n=2$ 215), quando se analisou a associação entre essas variáveis pelo teste qui-quadrado de Mantel \& Haenszel, o resultado não se revelou estatisticamente significativo $\left(\chi^{2} \mathrm{MH}=1,72\right.$, um grau de liberdade $p=0,1898)$.

Estudo realizado em Piracicaba, SP, com escolares da rede pública de ensino, também 
evidenciou que, conforme aumentava a idade, diminuía a proporção de alunos que consumiam a alimentação escolar com maior freqüência ${ }^{12}$.

Quando se analisou a "participação dos alunos no Programa", discriminando os escolares de acordo com o gênero ( $n=2$ 652), observou-se forte associação entre essas variáveis $\left(\chi^{2} \mathrm{MH}=8,13\right.$, um grau de liberdade significativo a $1 \%-p=0,0044)$, mesmo não sendo muito diferentes as proporções (85,5\% para os meninos e $81,3 \%$ para as meninas). No entanto, a associação do gênero com a "freqüência semanal de consumo da alimentação escolar" ( $n=2$ 215), não foi estatisticamente significativa, à semelhança dos resultados obtidos quando se analisou a influência da idade $(\mathrm{MH}=0,18$, com 1 grau de liberdade $-p=0,9100$ ).

As diferenças de consumo entre os jovens vêm sendo percebidas e mais intensamente exploradas, visando identificar as alterações no comportamento alimentar de acordo com o gênero e a faixa etária.

Estudo realizado pelo Instituto SODEXHO21 procurou analisar a diferenciação dos comportamentos alimentares, distinguindo-se os jovens de 5 a 17 anos por gênero em 11 países, inclusive no Brasil. Esse estudo mostrou que os jovens vêm adquirindo autonomia alimentar, tendência mais nítida entre os adolescentes, principalmente no ambiente escolar. Mostrou, também, a existência de dois modelos de alimentação que caracterizam a diferenciação por gênero e idade, quanto à ingestão de energia, à estrutura da refeição, ao tipo de alimento e à percepção no que diz respeito ao papel da alimentação.

$\mathrm{O}$ estudo de Heald ${ }^{22}$ mostrou que os meninos adolescentes americanos preferem alimentos com maiores teores de gordura, sal e açúcar, enquanto as meninas preferem os vegetais. $O$ autor destacou a influência da busca pela estética ideal como motivação para as meninas selecionarem seus alimentos. Kazapi et al. ${ }^{23}$, analisando o consumo de energia e macronutrientes de aproximadamente 800 escolares de Florianópolis, SC, observou que, em relação às meninas, os meninos ingeriam mais energia e proteínas e menos lipídeos.

Face ao exposto, é recomendável que os planejadores de programas de alimentação, visando um melhor atendimento do grupo alvo do Programa, levem em consideração que as preferências alimentares são influenciadas especialmente pelo gênero.

No presente estudo buscou-se, ainda, identificar a existência de associação entre o número de refeições que o aluno recebe no domicílio e o consumo da alimentação escolar.

O número de refeições constitui indicador importante do panorama alimentar e do padrão nutricional dos escolares. Os dados desta pesquisa evidenciaram que $15,0 \%$ a $20,0 \%$ dos escolares omitiam da sua pauta alimentar diária pelo menos uma refeição principal. Destaca-se que 19,5\% dos escolares declararam não consumir o café da manhã, enquanto $14,6 \%$ não almoçavam e $16,1 \%$ não jantavam.

É importante lembrar que a omissão de refeição é um fenômeno comum entre os adolescentes, sendo o desjejum a refeição mais negligenciada, seguida pelo jantar; freqüentemente, são substituídos por lanches com baixa densidade de nutrientes, muitas vezes ricos em gordura e açúcares e pobres em vitaminas, minerais e fibras ${ }^{24}$.

Os dados da presente pesquisa não captaram a associação $\left(\chi^{2} \mathrm{MH}=0,59\right.$, com 1 grau de liberdade $-p=0,4390)$ entre o número de refeições que o aluno recebe em casa e a variável "participação do aluno no Programa". No entanto, revelaram que a "freqüência de consumo de alimentação escolar" e essa variável $(n=2215)$ estão negativamente correlacionadas $\left(\chi^{2} \mathrm{MH}=3,26,1\right.$ grau de liberdade, significativo a $10 \%-p=0,0708)$. Dos que fazem até 3 refeições no domicílio, 56,3\% consomem a alimentação escolar 4 a 5 vezes por semana e entre aqueles que informaram ter mais de 3 refeições/dia no domicílio, apenas 51,9\% a consomem com similar freqüência.

Pesquisa realizada pelo MEC ${ }^{25}$ mostrou que a alimentação escolar tem função substitutiva 
de refeição do domicílio nitidamente maior, quando se consideram os escolares moradores das regiões menos desenvolvidas, enquanto, nas demais regiões, ela exerce um papel de suplementação. Evidenciou, também, que para os diretores de $40 \%$ das escolas públicas urbanas de ensino fundamental do país, a merenda foi considerada, em 1996, a principal refeição diária para a maioria dos alunos sob a responsabilidade dos mesmos. Quando se consideraram as informações regionais para análise, 56\% e 50\% dos diretores entrevistados das Regiões Norte e Nordeste, respectivamente, forneceram essa resposta.

Não foi analisada na presente pesquisa a associação entre as variáveis "número de refeições que o aluno recebe na casa" e "renda familiar per capita dos escolares". No entanto, provavelmente os escolares com menor número de refeições realizadas no domicílio pertenciam às classes de menor renda familiar, e buscavam suprir uma deficiência consumindo a refeição ou alimentos distribuídos gratuitamente na escola. De acordo com o estudo realizado por Dall'Acqua ${ }^{26}$ em Campinas, SP, do total de crianças entrevistadas, cerca de $36 \%$ da faixa de renda familiar até 2,5 salários mínimos não tomavam desjejum.

No que se refere ao estado nutricional dos escolares, observou-se forte associação $\left(\chi^{2} \mathrm{MH}=27,33,1\right.$ grau de liberdade, significativo a $1 \%$ - $p<0,0001)$ entre a "participação do aluno no Programa" ( $n=2$ 657) e a condição de baixo peso. Buscou-se identificar a possível associação entre a situação nutricional e a "freqüência semanal de consumo da alimentação escolar" $(n=2$ 215) e os dados, apresentados na Tabela 2, evidenciam que a adesão semanal também é maior entre os escolares com baixo peso. O qui-quadrado, que acompanha a Tabela, mostra a forte associação (ao nível de 1\%) entre essas variáveis.

Deve-se destacar que a adesão diária de aproximadamente $50 \%$ dos escolares classificados abaixo do 5o Percentil (IMC) não pode ser considerada satisfatória. Considerando-se o elevado custo operacional do Programa e a expectativa dos governantes em relação à contribuição do mesmo para a melhoria do estado nutricional dos alunos, principalmente daqueles pertencentes às classes com menores rendimentos per capita, esse resultado mostra-se aquém do esperado.

Partindo-se do pressuposto que variáveis relativas às condições socioeconômicas possam afetar o consumo da refeição oferecida na escola, utilizou-se, como variável explanatória, a renda familiar per capita. Utilizou-se, também, a escolaridade da mãe que pode atuar indiretamente sobre essa variável, na medida em que ela proporciona maiores chances de participação no mercado de trabalho, e de receber maiores salários.

Constatou-se, neste trabalho, a forte associação negativa e estatisticamente significativa $\left(\chi^{2} \mathrm{MH}=123,89,1\right.$ grau de liberdade, valor $p<0,0001)$ entre as variáveis, "participação do aluno no Programa" e renda familiar per capita $(n=1805)$. Essa mesma tendência foi verificada

Tabela 2. Proporção de escolares segundo a categoria do estado nutricional com base no Índice de Massa Corporal e a freqüência semanal de consumo da alimentação escolar, 1997.

\begin{tabular}{|c|c|c|c|c|c|c|}
\hline \multirow[b]{2}{*}{ Categorias do estado nutricional } & \multicolumn{2}{|c|}{$1 \mathrm{vez}$} & \multicolumn{2}{|c|}{2 a 3 vezes } & \multicolumn{2}{|c|}{4 a 5 vezes } \\
\hline & $\mathrm{n}$ & $\%$ & $\mathrm{n}$ & $\%$ & $\mathrm{n}$ & $\%$ \\
\hline$<5$ ㅇ $P$ & 12 & 13,6 & 30 & 34,9 & 44 & 51,2 \\
\hline$\geq 5 \circ \mathrm{P}$ e $<10 \circ \mathrm{P}$ & 4 & 4,4 & 30 & 33,3 & 56 & 62,2 \\
\hline$\geq 10 \circ \mathrm{P}$ e $<85 \circ \mathrm{P}$ & 158 & 9,6 & 539 & 32,6 & 958 & 57,9 \\
\hline$\geq 85^{\circ} \mathrm{P}$ e $<95^{\circ} \mathrm{P}$ & 41 & 15,8 & 102 & 39,4 & 116 & 44,8 \\
\hline$\geq 95 \circ P$ & 20 & 16,0 & 53 & 42,4 & 52 & 41,6 \\
\hline
\end{tabular}

$\chi^{2}$ de Mantel \& Haenszel $=28,62$ com um grau de liberdade, valor $p<0,0001$ 
quando se analisou a associação dessa variável com a "freqüência semanal de consumo da alimentação escolar" ( $n=1$ 514). Assim, à medida que aumenta a renda familiar per capita, a percentagem de alunos que revelam consumir a alimentação escolar com maior freqüência tende a diminuir. O resultado do qui-quadrado, que acompanha a Tabela 3, mostra a forte associação (1\%) entre essas variáveis. Resultados semelhantes foram observados por Silva et al. ${ }^{12}$, em trabalho envolvendo amostra de escolares da rede pública de ensino de Piracicaba, SP.

A associação captada na presente pesquisa confirma a importância do Programa para as famílias de baixa renda, de acordo com o que foi verificado em pesquisa realizada por Dall'Acqua ${ }^{26}$. Nesse estudo verificou-se que cerca de $70 \%$ das famílias de escolares da rede pública de ensino de Campinas, SP, com renda familiar até US\$170.00, declarou que a alimentação escolar era importante na decisão de mandar seus filhos à escola.

Observa-se, ainda, na Tabela 3, que a maior freqüência de consumo de merenda (quatro ou cinco vezes por semana) é observada entre os escolares pertencentes ao estrato de menor renda. A proporção supera em 24 pontos percentuais a verificada entre os alunos mais ricos (estrato de maior renda). No entanto, mesmo entre os alunos classificados nos três estratos de menores rendimentos, a adesão mostra-se entre $60 \%$ e $70 \%$.
No final da década de 80, verificou-se também, por meio dos dados da PNSN, que havia redução do consumo efetivo da merenda, conforme o aumento da renda familiar das crianças. Entretanto, é importante lembrar que, na época dessa pesquisa, os alimentos tradicionais, freqüentemente preferidos pelos escolares, apenas integravam de forma modesta o $\mathrm{PNAE}^{7}$. Isto sugere que a não aceitação das preparações oferecidas na escola estaria refletindo uma rejeição voluntária às preparações distribuídas na época.

Pesquisa realizada por Brandão ${ }^{13} \mathrm{em}$ dez unidades escolares municipais de Campinas, SP, entre 1997 a 1999, também revelou que, mesmo passando por restrições econômicas, as crianças não participavam do Programa. Nesse estudo, a percentagem de adesão para as crianças de nível socioeconômico baixo e muito baixo situava-se entre 49\% e 52\%. É importante notar que esse autor, a partir de análise de correlação, demonstrou que os baixos índices de participação das crianças junto ao Programa de Merenda Escolar não dependiam exclusivamente da renda familiar dos estudantes, devendo existir outros fatores que influenciavam de forma mais expressiva tal comportamento.

O nível de escolaridade do responsável pela família, além de bom indicador do nível socioeconômico das famílias, é uma informação mais facilmente obtida e menos sujeita a imprecisão, ao ser fornecida pelo entrevistado. De acordo com Monteiro \& Szarfac ${ }^{27}$, a escolaridade, invariavelmente, contribui para o aumento das possibilidades de trabalho e salário.

Tabela 3. Proporção de escolares segundo estratos de renda familiar per capita (RFPC) e a freqüência semanal de consumo da alimentação escolar, 1997.

\begin{tabular}{|c|c|c|c|c|c|c|}
\hline \multirow[b]{2}{*}{ Estrato de RFPC (R\$) } & \multicolumn{2}{|c|}{$1 \mathrm{vez}$} & \multicolumn{2}{|c|}{2 a 3 vezes } & \multicolumn{2}{|c|}{4 a 5 vezes } \\
\hline & $\mathrm{n}$ & $\%$ & $\mathrm{n}$ & $\%$ & $\mathrm{n}$ & $\%$ \\
\hline $0 \vdash 25$ & 12 & 5,4 & 58 & 25,9 & 154 & 68,7 \\
\hline $25 \vdash 50$ & 28 & 8,3 & 105 & 31,1 & 205 & 60,6 \\
\hline $50 \vdash 75$ & 19 & 7,1 & 90 & 33,7 & 158 & 59,2 \\
\hline $75 \vdash 100$ & 22 & 11,6 & 66 & 34,9 & 101 & 53,4 \\
\hline $100 \vdash 150$ & 25 & 12,3 & 71 & 34,8 & 108 & 52,9 \\
\hline$>150$ & 54 & 18,5 & 109 & 37,3 & 129 & 44,2 \\
\hline
\end{tabular}

$\chi^{2}$ de Mantel \& Haenszel $=46,21$ com um grau de liberdade, valor $p<0,0001$. 
Quando se analisou a influência da escolaridade da mãe na participação do aluno no Programa" ( $n=2$ 187), observou-se forte associação entre as variáveis $\left(\chi^{2} \mathrm{MH}=41,59,1\right.$ grau de liberdade, valor $p<0,0001)$.

Os dados da Tabela 4 mostram a mesma tendência entre as variáveis consideradas, ou seja, entre a escolaridade da mãe e a "freqüência semanal de consumo" ( $n=1816)$ a partir dos resultados do teste qui-quadrado (significativo ao nível de 1\%). À semelhança da renda, à medida que cresce a escolaridade da mãe se tende a reduzir a freqüência semanal de consumo da alimentação oferecida na escola. Neste sentido, nota-se que a freqüência de consumo da alimentação oferecida na escola, dos escolares cujas mães têm de 1 a 4 anos de escolaridade, é 22,8 pontos percentuais superior à encontrada para os escolares cujas mães têm de 12 a 15 anos de escolaridade.

Oliveira et al. ${ }^{28}$ também verificaram que o consumo da merenda escolar é inversamente proporcional ao nível de escolaridade dos responsáveis pelos alunos. Esses autores concluíram, ainda, que embora crianças de famílias de baixa escolaridade consumam com maior freqüência a merenda escolar, a menor adesão às preparações parece estar condicionada aos hábitos alimentares ou à monotonia dos cardápios, ou ainda, à qualidade dos alimentos que os compõem.

Quanto à influência da presença de cantina escolar na unidade de ensino sobre a "participação do aluno no Programa", verificou-se correlação negativa $\left(\chi^{2}=308,53\right.$ e $p<.0001$, significativa a $1 \%$ ) entre tais variáveis ( $n=2652$ ). É importante destacar que, na época da pesquisa, nove entre as dez unidades de ensino que compunham a amostra mantinham esse tipo de serviço (unidades dos municípios de Tailândia, PA, Contagem, MG, Baldim, MG e dos dois municípios de Santa Catarina). Cerca de $70 \%$ dos alunos que afirmaram não participar do Programa freqüentavam essas unidades de ensino.

O grau de influência exercida pela presença da cantina escolar na unidade de ensino sobre a "freqüência de consumo da alimentação escolar" ( $n=2215)$ também foi analisado. Verificou-se uma forte associação negativa entre essas variáveis $\left(\chi^{2}=125,19 p<.0001\right.$ para dados de escolas com cantina e $\chi^{2}=694,79 p<.0001$ para sem cantina). A proporção de alunos que consumiam com maior freqüência a alimentação gratuita, distribuída na escola, foi de $66,1 \%$ nas unidades sem cantina; ou seja, cerca de 25 pontos percentuais acima da proporção observada nas unidades escolares em que funcionavam cantinas comerciais.

Silva et al. ${ }^{12}$, analisando o consumo de merenda escolar em Piracicaba, SP, observaram que $35,6 \%$ das crianças a consumiam, enquanto $76,1 \%$ costumavam adquirir alimentos na cantina escolar.

Esses resultados são preocupantes, uma vez que a preferência dos escolares recaem sobre os alimentos de baixa qualidade nutricional ${ }^{29}$, os quais, se consumidos em excesso, a curto prazo

Tabela 4. Proporção de escolares segundo a escolaridade da mãe e a freqüência semanal de consumo da alimentação escolar, 1997.

\begin{tabular}{|c|c|c|c|c|c|c|}
\hline \multirow{2}{*}{ Escolaridade da Mãe (anos) (semanal) } & \multicolumn{2}{|c|}{$1 \mathrm{vez}$} & \multicolumn{2}{|c|}{2 a 3 vezes } & \multicolumn{2}{|c|}{4 a 5 vezes } \\
\hline & $\mathrm{n}$ & $\%$ & $n$ & $\%$ & $n$ & $\%$ \\
\hline Nenhuma & 1 & 5,9 & 3 & 17,7 & 13 & 76,5 \\
\hline $1 \vdash 4$ & 62 & 8,3 & 264 & 35,5 & 417 & 56,1 \\
\hline $5 \vdash 8$ & 71 & 10,8 & 219 & 33,3 & 368 & 55,9 \\
\hline $9 \vdash 11$ & 53 & 15,5 & 114 & 33,2 & 176 & 51,3 \\
\hline $12 \vdash 15$ & 10 & 19,2 & 23 & 44,2 & 19 & 36,5 \\
\hline$>15$ & 1 & 33,3 & 1 & 33,3 & 1 & 33,3 \\
\hline
\end{tabular}

$\chi^{2}$ de Mantel $\&$ Haenszel $=14,7$ com um grau de liberdade, valor $p<0,0002$. 
podem acarretar problemas, entre os quais destaca-se a obesidade, além dos distúrbios cardiovasculares e a hipertensão, na fase adulta.

Visando analisar simultaneamente a influência das diversas variáveis consideradas (idade, gênero, estado nutricional, número de refeições realizadas no domicílio, freqüência de consumo de alimentos adquiridos na cantina escolar, renda familiar per capita e escolaridade dos pais) no consumo da alimentação escolar, adotou-se o modelo de lógite, para cada uma das variáveis dependentes, ou seja, "participação do aluno no Programa" e "freqüência semanal de consumo da alimentação escolar". As estimativas dos coeficientes das equações ajustadas podem ser observadas na Tabela 5.

Os dados da Tabela 5 mostram que, no modelo ajustado para a variável dependente "participação do aluno no Programa", algumas variáveis explanatórias, como a renda familiar per capita e escolaridade dos pais, apresentam sinal negativo, indicando associação inversa: ou seja, descontada a influência das demais variáveis, aumentando-se a renda familiar per capita e a escolaridade dos pais, diminui a participação do aluno no Programa.

Em relação a esses resultados, observou-se também que, no modelo lógite multinomial cumulativo, as mesmas variáveis discutidas acima apresentam sinal positivo, indicando associação positiva com a variável dependente "freqüência semanal de consumo de alimentação escolar" , o que seria, aparentemente incoerente. Entretanto, é necessário destacar que o lógite acumulou as menores freqüências de consumo da alimentação escolar (uma vez por semana e duas ou três vezes por semana), ou seja, modelou a proporção dos alunos que a consomem com menor freqüência, justificando o sinal invertido. Então, conforme aumentam a renda familiar per capita e a escolaridade dos pais, cresce a proporção de crianças que consomem a alimentação escolar com menor freqüência.

De um modo geral, os resultados dos modelos de lógite confirmam as associações entre

Tabela 5. Estimativas dos coeficientes das equações dos modelos de lógite ajustados.

\begin{tabular}{|c|c|c|c|c|c|c|c|c|c|c|c|c|}
\hline \multirow{6}{*}{$\begin{array}{c}\text { Variáveis } \\
\text { explanatórias }\end{array}$} & \multicolumn{12}{|c|}{ Variáveis dependentes } \\
\hline & \multicolumn{6}{|c|}{ "Participação do aluno no Programa" } & \multicolumn{6}{|c|}{ "Freqüência de consumo da alimentação escolar" } \\
\hline & \multicolumn{6}{|c|}{ Parâmetros estimados } & \multicolumn{6}{|c|}{ Parâmetros estimados } \\
\hline & & & & Sub-amo & ostras & & & & & Sub-amo & ostras & \\
\hline & \multicolumn{2}{|c|}{$\begin{array}{l}\text { Amostra total } \\
\quad(n=1488)\end{array}$} & \multicolumn{2}{|c|}{$\begin{array}{c}\text { Com cantina } \\
\text { escolar }(n=547)\end{array}$} & \multicolumn{2}{|c|}{$\begin{array}{c}\text { Sem cantina } \\
\text { escolar }(n=1111)\end{array}$} & \multicolumn{2}{|c|}{$\begin{array}{l}\text { Amostra total } \\
\quad(n=1243)\end{array}$} & \multicolumn{2}{|c|}{$\begin{array}{c}\text { Com cantina } \\
\text { escolar }(n=444)\end{array}$} & \multicolumn{2}{|c|}{$\begin{array}{c}\text { Sem cantina } \\
\text { escolar }(n=258)\end{array}$} \\
\hline & $\beta$ & $\mathrm{NF}^{\mathrm{a}}$ & $\beta$ & $\mathrm{NF}^{\mathrm{a}}$ & $\beta$ & $\mathrm{NF}^{\mathrm{a}}$ & $\beta$ & $\mathrm{NF}^{\mathrm{a}}$ & $\beta$ & $N F^{a}$ & $\beta$ & $\mathrm{NF}^{\mathrm{a}}$ \\
\hline \multirow{3}{*}{$\begin{array}{l}\text { Intercepto }\left(\alpha_{1}\right) \\
\text { Intercepto }\left(\alpha_{2}\right) \\
\text { Idade }\end{array}$} & 5,4007 & $<0,0001^{* *}$ & 3,9803 & $<0,0001^{* *}$ & 6,1162 & $<0,0001^{* *}$ & $-3,4476$ & $<0,0001^{* *}$ & $-2,2745$ & $0,0002^{* *}$ & $-3,0067$ & $<0,0001^{* *}$ \\
\hline & & & & & & & $-1,5345$ & 0,0001 ** & $-0,1759$ & 0,7722 & $-0,962$ & $<0,0001^{* *}$ \\
\hline & $-0,0155$ & $<<0,0001^{* *}$ & $-0,0230$ & $<0,0001 * *$ & $-0,0122$ & $0,0010^{* *}$ & 0,0043 & $0,0474^{*}$ & 0,0085 & $0,0217^{*}$ & - & - \\
\hline Gênero & $-0,5064$ & A $0,0009 * *$ & - & - & - & - & - & - & - & - & - & - \\
\hline Percentil do IMC & - & - & - & - & $-0,0068$ & 0,0640 & 0,0068 & $0,0003^{* *}$ & - & - & 0,0066 & $0,0014^{\star *}$ \\
\hline $\begin{array}{l}\mathrm{N}^{\circ} \text { de refeições } \\
\text { domicílio }\end{array}$ & - & - & - & - & $-0,3092$ & $0,0068^{* *}$ & - & - & - & - & - & - \\
\hline RFPC $^{\mathbf{d}}$ & $-0,0032$ & $<0,0001$ ** & $-0,0022$ & $0,0024^{*}$ & - & - & 0,0021 & $<0,0001 * *$ & 0,0022 & $<0,0023^{* *}$ & - & - \\
\hline Escolaridade pai & $-0,0959$ & $<0,0001^{* *}$ & $-0,1206$ & $0,0001 * *$ & - & - & 0,0402 & $0,0277^{* *}$ & - & - & - & - \\
\hline Escolaridade mãe & - & - & - & - & $-0,1040$ & $0,0012^{* *}$ & - & - & 0,0728 & $0,0048^{* *}$ & - & - \\
\hline $\begin{array}{l}\text { Consumo } \\
\text { cantina escolar }\end{array}$ & - & - & 0,5455 & $0,0001^{* *}$ & - & - & - & - & $-0,4357$ & $0,0013^{* *}$ & - & - \\
\hline $\begin{array}{l}\text { Índice de } \\
\text { concordânciab }\end{array}$ & 73,1 & & 75,3 & & 65,3 & & 59,7 & & 64,9 & & 52,6 & \\
\hline D de Sommer ${ }^{\mathbf{b}}$ & 0,468 & & 0,510 & & 0,319 & & 0,212 & & 0,305 & & 0,106 & \\
\hline
\end{tabular}

Os traços (-) indicam que a variável não faz parte do modelo; ${ }^{a}$ NS= Nível de significância: **significativo a 1\% e *significativo a 5\%; ${ }^{\mathbf{b}}$ Estatísticas que mostram a correlação entre a probabilidade estimada e a resposta observada; ${ }^{C} \mathrm{IMC}=$ Índice de Massa Corporal; ${ }^{\mathbf{d}}$ Renda familiar per capita. 
as variáveis captadas pelos testes de qui-quadrado (que acompanham as tabelas de contingências) e as considerações anteriormente apresentadas.

Quando se analisam os dados da Tabela 5, tendo por base o total de alunos, observa-se que as variáveis renda familiar per capita, escolaridade do pai, idade e estado nutricional dos alunos, estão inversamente associadas à "freqüência semanal de consumo da alimentação escolar" ( $n=1$ 243). Verifica-se, também, quanto à variável "gênero", que ser menina afeta negativamente a "participação do aluno no Programa" ( $n=1488)$.

Ainda de acordo com a Tabela 5, quando se analisa o grupamento de alunos pertencentes às unidades escolares onde existem estabelecimentos que comercializam alimentos $(n=547)$, observa-se que afetam negativamente a "participação do aluno no Programa" os fatores: maiores rendimentos familiares, maior escolaridade do pai, maior freqüência semanal de consumo de alimentos na cantina escolar e maior idade dos alunos. Essas mesmas variáveis são inversamente associadas à "freqüência semanal de consumo da alimentação escolar", com exceção da "idade do aluno" ( $n=444)$.

Quanto ao grupamento de alunos pertencentes às unidades de ensino que não dispõem de cantina escolar, verifica-se que a "participação do aluno no Programa" está inversamente associada à escolaridade da mãe, ao número de refeições realizadas no domicílio, à idade dos alunos e ao estado nutricional $(n=1111)$. Tanto a situação nutricional como o número de refeições recebidas no domicílio guardam forte relação com o nível socioeconômico da família. No entanto, essas variáveis foram consideradas não colineares à renda familiar per capita e à escolaridade dos pais (tendo por base as análises dos testes estatísticos). Além disso, apresentaram elevada significância de associação.

Ainda em relação ao grupamento de alunos pertencentes às unidades de ensino sem os serviços de cantina, na Tabela 5 verifica-se que o estado nutricional é a única variável inversamente associada à "freqüência semanal de consumo da alimentação escolar". Deve-se salientar que essas unidades escolares abrigavam substancial parcela dos alunos com menor renda familiar per capita, cujos pais possuíam poucos anos de estudo ${ }^{14}$. Tais características revelam as condições socioeconômicas desfavoráveis em que vivem as famílias. Esse fato pode justificar o resultado, em que apenas o coeficiente do estado nutricional se revelou estatisticamente significativo.

Os resultados obtidos neste estudo mostram a necessidade de analisar, de forma pormenorizada, os motivos da recusa voluntária dos escolares à alimentação escolar. Note-se que a refeição oferecida na escola visa manter a criança alimentada durante a jornada de aula, independente da sua condição socioeconômica. A preferência de grande parte dos escolares pelos alimentos, nem sempre saudáveis, comercializados nas cantinas, é uma constatação preocupante diante da prevalência da obesidade, que aumenta entre os adolescentes, nas últimas décadas, no Brasili ${ }^{30}$.

Por outro lado, os resultados evidenciam a importância da contribuição nutricional do Programa para os escolares com déficit de peso e pertencentes às famílias de nível socieconômico mais baixo. Embora o objetivo do Programa não seja erradicar a desnutrição, torna-se imprescindível o oferecimento de refeições nutricionalmente balanceadas e/ou fortalecidas, com o intuito de, pelo menos, diminuir o impacto negativo das carências nutricionais.

\section{O N CLUS Ã O}

De um modo geral, pode-se inferir que a adesão ao Programa de Alimentação Escolar, considerada como a freqüência diária de consumo da refeição gratuita oferecida na escola, é baixa, além de ser fortemente afetada pelas variáveis socioeconômicas, idade e estado nutricional dos alunos. Optam pela alimentação gratuita os alunos mais novos, os que revelam algum tipo de comprometimento nutricional, os pertencentes aos 
estratos mais baixos de renda familiar per capita e aqueles cujos pais possuem menor escolaridade.

Nas unidades de ensino que não dispõem de estabelecimentos que comercializam alimentos, há maior freqüência de consumo da alimentação escolar, sendo esta condicionada somente pela variável "estado nutricional" do aluno. Tais resultados sugerem que o Programa, mesmo sendo concebido para ser universal, na prática, influenciado pelas características de seus usuários, assume caráter focalizado, beneficiando quase que somente os escolares com déficit de peso.

Os resultados relatados parecem demonstrar que os problemas de adesão constatados em 1989 pela PNSN persistem, mesmo após a descentralização do Programa. Portanto, a sugestão apresentada na época pelo referido órgão, de que "é desejável que os dados sejam analisados no sentido de melhorar a eficiência desse tipo de assistência e não como justificativa para possíveis cortes nos investimentos governamentais na área", deve ser considerada.

Espera-se que o presente estudo possa contribuir com os formuladores de políticas públicas, no delineamento ou reformulação do Programa, com vistas a promover a adequação do seu atendimento às características dos beneficiários e, conseqüentemente, aumentar a sua aceitação. Espera-se também, que constitua subsídio para pesquisas futuras, cujo objetivo seja identificar os motivos da rejeição da alimentação escolar por expressiva parcela da população alvo.

Recomenda-se a realização de pesquisas sobre a preferência dos alunos pelos distintos tipos de alimentos e formas de preparação, assim como pesquisas relacionadas à estrutura de distribuição e horário das refeições, visando a melhoria dos resultados observados.

\section{RE FER Ê N CIAS}

1. Fundo Nacional de Desenvolvimento da Educação. Relatório de atividades [Internet]. Brasília: MEC; 2003. Disponível em: http://www.fnde.gov.br/ Programas/Merenda
2. Tribunal de Contas da União. Auditoria operacional nos programas de suplementação alimentar no Governo Federal. Brasília: TCU; 1993.

3. Peliano AMTM, coordenador. Um balanço das ações de governo no combate à fome e à miséria [apostila]. Brasília: IPEA; 1993. 63p.

4. Instituto Nacional de Alimentação e Nutrição. Pesquisa Nacional de Saúde e Nutrição: resultados preliminares [mimeografado]. Brasília;1990.

5. Peliano AM. Quem se beneficia dos Programas Governamentais de Suplementação Alimentar [textos para discussão, 205]. Brasília: Instituto de Pesquisas Econômicas Aplicadas; 1990.

6. Lopes JRB, Telles SMS. Caracterização das populações pobres no Brasil e de seu acesso a programas sociais. In: Galeazzi MAM, organizador. Segurança alimentar e cidadania. Campinas: Mercado de Letras; 1996. p.63-92.

7. Silva MV, Ometto $A M H$, Oetterer M, Sturion GL, Pipitone MAP, Furtuoso MCO. Escolares brasileiros: análise do estado nutricional e do consumo de merenda segundo diferenças regionais e entre classes de renda. In: Anais do 16 Congresso Brasileiro de Ciência e Tecnologia de Alimentos; Rio de Janeiro; 1998. Rio de Janeiro: SBCTA; 1998a. p. 635-8.

8. Ometto AHO, Furtuoso COM, Silva MV, Oetterer M, Pipitone MAP, Sturion GL. Acesso aos Programas de Suplementação Alimentar: desigualdades regionais e setoriais. Saúde em Revista. 2001; 3(5/6):23-31.

9. Pipitone MAP. Programa de Alimentação Escolar: um estudo sobre descentralização, escola e educadores [tese]. Campinas: Universidade Estadual de Campinas; 1997.

10. Spinelli MAS. Alimentação Escolar: da centralização à descentralização [tese]. Campinas: Universidade Estadual de Campinas; 1997.

11. Vieira MNCM. A prática do Programa de Alimentação Escolar do Município de Ribeirão Preto mediante a percepção dos usuários [dissertação]. Ribeirão Preto: Universidade de São Paulo; 1997.

12. Silva MV, Pipitone MAP, Sturion GL, Caroba DCR. Educação e saúde e sua relação com o estado nutricional e práticas alimentares de escolares de $1^{\circ} \mathrm{Grau}$. In: Anais do 16을 Congresso Brasileiro de Ciência e Tecnologia de Alimentos; Rio de Janeiro; 1998. Rio de Janeiro: SBCTA; 1998b. p.616-9.

13. Brandão TM. Avaliação da aceitação e preferências de cardápios do Programa de Merenda Escolar em escolas municipais do ensino fundamental da cidade de Campinas [dissertação]. Campinas: Universidade Estadual de Campinas; 2000. 
14. Sturion GL. Programa de Alimentação Escolar: avaliação do desempenho em dez municípios brasileiros [tese]. Campinas: Universidade Estadual de Campinas; 2002.

15. Ministério da Educação e do Desporto. Avaliação do impacto distributivo e elaboração de sistemática de monitoramento do PNAE [relatório final de pesquisa]. Brasília: MEC; 2002.

16. World Health Organization. Physical Status: The use and interpretation of anthropometry. Geneva: WHO; 1995. Technical Report Series, 854.

17. Center for Disease Control and Prevention, National Center for Health Statistics. CDC/NCHS. 2000 CDC Growth charts for the United States: Methods and development [series 11,246]. Washington, DC:; 2002. 190p.

18. Mantel M, Haenszel W. Statistical aspects of analysis of data from retrospective studies of disease. J Natl Center Inst. 1959; 22 (Special): 719-48.

19. Allison PD. Logistic regression using the SAS System: Theory and application. Cary: SAS Institute; 1999.

20. Freund RJ, Littell RC. SAS System for regression: SAS series in statistical applications. 2nd ed. Cary: SAS Institute; 1991.

21. Instituto SODEXHO. Instituto para o Desenvolvimento da Qualidade de Vida no Cotidiano. Masculino e feminino: os diferentes hábitos alimentares entre crianças e jovens. São Paulo; 2001.

22. Heald FP. Fast food and snack food: beneficial or deleterious. J Adolesc Health. 1992; 13(Special): 380-3.

23. Kazapi IM, Di Pietro F, Avancini SRP, Freitas SFT, Tramonte VLCG. Consumo de energia e macronutrientes por adolescentes de escolas públicas e privadas. Rev Nutr. 2001; 14(Supl.):27-33.
24. Vieira VCR, Priore SE. Hábitos alimentares de adolescentes. Rev Nutr. 2001; 14-20.

25. Ministério de Educação e do Desporto, Instituto Nacional de Estudos e Pesquisas Educacionais. Relatório de avaliação da descentralização de recursos do FNDE e da merenda escolar: resultados preliminares. Campinas: NEPPA/Unicamp; 1998b. p.82-129.

26. Dall'Acqua FM. O ajustamento econômico e as políticas sociais: o caso do Programa de Merenda Escolar. In: Campino ACC, Amaral CM, organizadores. Questões sociais no Brasil. São Paulo: USP; 1994. p.57-77.

27. Monteiro CA, Szarfarc S. A alimentação. In: Monteiro CA, organizador. Saúde e nutrição das crianças de São Paulo: diagnóstico, contrastes sociais e tendências. São Paulo: Hucitec; 1988. p.71-81.

28. Oliveira J, Cyrillo DC, Lajolo FM. O consumo da merenda escolar nas escolas municipais de São Paulo. In: Livros de Resumos do 4ํㅡㄹ Congresso Nacional de Alimentação e Nutrição; 1996. São Paulo: SBAN; 1996. p.24.

29. Sturion GL, Silva MV, Ometto AMO, Pipitone MAP, Furtuoso COM. Freqüência de consumo da alimentação escolar e preferências dos alunos. In: Anais do 5o Simpósio Latino Americano de Ciência de Alimentos; [CD-Rom]; Campinas, 2003. Campinas: Unicamp; 2003.

30. Caroba DCR. A escola e o consumo alimentar de adolescentes matriculados na rede pública de ensino [dissertação]. Piracicaba: Escola Superior de Agricultura Luiz de Queiroz da Universidade de São Paulo; 2002

Recebido para publicação em 29 de julho de 2003 e aceito em 5 de maio de 2004. 